\title{
Antimicrobial and osteogenic effect of Ag-implanted titanium with a nanostructured surface
}

This article was published in the following Dove Press journal:

International Journal of Nanomedicine

17 February 2012

Number of times this article has been viewed

\section{Yanhua Zheng' \\ Jinbo $\mathrm{Li}^{2}$ \\ Xuanyong Liu $^{2}$ \\ Jiao Sun'}

'Shanghai Biomaterials Research and Testing Center, Shanghai Key Laboratory of Stomatology, Ninth People's Hospital, Shanghai Jiaotong University School of Medicine, Shanghai 2000II, People's Republic of China; ${ }^{2}$ State Key Laboratory of High Performance Ceramics and Superfine Microstructure, Shanghai Institute of Ceramics, Chinese Academy of Sciences, Shanghai 200050, People's Republic of China
Correspondence: Xuanyong Liu Shanghai Institute of Ceramics, Chinese Academy of Sciences, No 1295

Ding-xi Road, Shanghai, China, 200050

Tel +862152412409

Fax +86 2I 524I 2409

Email xyliu@mail.sic.ac.cn

Jiao Sun

Ninth People's Hospital, Shanghai Jiaotong University School of Medicine, No 639

Zhi-zaoju Road, Shanghai, China, 2000I I

Tel +86 2l 63034903

Fax +8621630II643

Email jiaosun59@yahoo.com

\begin{abstract}
Ag-implanted titanium with a nanostructured surface was prepared by hydrothermal treatment with $\mathrm{H}_{2} \mathrm{O}_{2}$ followed by Ag plasma immersion ion implantation. Streptococcus mutans, Porphyromonas gingivalis and Candida albicans were chosen for antimicrobial tests. Genes related to microbial structure or adhesion, namely glucan-binding proteins B $(G b p B)$, fimbria protein A (FimA), and agglutinin-like sequence4 ( $\left.A l_{s} 4\right)$, were examined. The osteoblast's attachment, viability, and quantitative analysis of osteogenic gene expression (Alp, Ocn, RunX2) on titanium surfaces were evaluated. Scanning electron microscopy (SEM) revealed that $\mathrm{Ag}$ nanoparticles of approximately $10 \mathrm{~nm}$ were incorporated on the nanostructured surface of titanium after Ag plasma immersion ion implantation. Trials showed that $93.99 \%$ of S. mutans, $93.57 \%$ of $P$. $g$, and $89.78 \%$ of C. albicans were killed on the Ag-implanted titanium with a nanostructured surface. Gene expressions from the three microorganisms confirmed the antimicrobial activities of the Ag-implanted titanium with a nanostructured surface. Furthermore, the adhesive images and viability assays indicated that the Ag-implanted titanium with a nanostructured surface did not impair osteoblasts. The expressions of osteoblast phenotype genes in cells grown on the Ag-implanted titanium surface were significantly increased. The results of this study suggest that the Ag-implanted titanium with a nanostructured surface displays good antimicrobial properties, reducing gene expressions of microorganisms, and excellent cell adhesion and osteogenic effects.
\end{abstract}

Keywords: titanium, nanostructured, silver, antimicrobial, osteogenic

\section{Introduction}

Infections associated with dental implants are a challenge to the long-term "survival" rate of implants and may cause the treatment to fail. ${ }^{1,2}$ The titanium surfaces of implants are suitable for microbial colonization and biofilm formation. ${ }^{3}$ Furthermore, there are limitations to the use of ultraclean surgical techniques and prophylactic antibiotics, and dental implants not only penetrate bone but also gingiva, which means that the transgingival abutment of the implant can be an important portal of microbial entry. Therefore, strategies involving the delivery or incorporation of antibiotics such as gentamicin and vancomycin on some titanium implants have been tried to reduce infections. ${ }^{4,5}$ With increasing concerns over antibiotic-resistant pathogens, however, non-conventional antibiotics have been developed in an attempt to avoid these problems without compromising the treatment of infection. Silver is effective against a broad spectrum of bacterial and fungal species, including strains that are resistant to antibiotics. ${ }^{6}$ Silver nanoparticles are considered to be even more active due to their large surface area to volume ratio. ${ }^{7}$ Previous work has indicated that silver nanoparticles 
embedded in titanium may be highly effective in inhibiting both Staphylococcus aureus and Escherichia coli. ${ }^{8}$ However, these strains are not representative of oral bacteria - dental implants may face different microbial strains, especially anaerobes or facultative anaerobes. During implantation, Streptococcus mutans (S. mutans), which makes up the majority of oral bacteria within saliva, pose a high risk of contaminating surgical sites. Porphyromonas gingivalis $(P g)$, which is likely to the subgingival flora of chronic periodontitis, were detected at elevated levels in peri-implant diseases of dental implants. ${ }^{9}$ Candida albicans (C. albicans), which has a high potential to adhere to implant surfaces as well as oral tissues, is often involved in oral infections. ${ }^{10}$ Therefore it is important to evaluate the antimicrobial properties of dental implanted materials against $S$. mutans, $P g$, and $C$. albicans.

However, if titanium surfaces that have been implanted with silver fail to support osteogenesis then any antimicrobial properties may remain insignificant. It is believed that proper nanotopography can enhance osteogenesis from the biomimetic viewpoint since bone tissues are composed of hierarchical nanocomposites. ${ }^{11,12}$ Hence, it was hypothesized that a nanostructured surface implanted with silver nanoparticles could possess relatively good antimicrobial properties and simultaneously enhance tissue cells (such as osteoblasts). In the present study, silver-implanted titanium materials with a nanostructured surface were compared with untreated titanium and $\mathrm{H}_{2} \mathrm{O}_{2}$-treated titanium. The in vitro antimicrobial activities of these materials against $S$. mutans, $P g$, and C. albicans were assessed and their antimicrobial mechanisms were investigated at the genetic level. Moreover, the in vitro osteogenic effect of the surfaces was evaluated by observing the attachment, viability, and osteogenic gene expression using mouse calvaria-derived osteoblast cells.

\section{Materials and methods}

\section{Preparation and characterization of titanium samples}

Commercial pure Ti plates (Grade 2) with dimensions of $10 \mathrm{~mm} \times 10 \mathrm{~mm} \times 1 \mathrm{~mm}$ were ultrasonically cleaned several times in ethanol and deionized water, followed by soaking in a $5 \mathrm{wt} \%$ oxalic acid solution at $100^{\circ} \mathrm{C}$ for 2 hours to remove the oxide layer and obtain a homogeneous rough surface. Then the samples were ultrasonically cleaned in deionized water and dried in the ambient atmosphere. Each pretreated Ti plate was immersed in a solution of $5 \mathrm{~mL} \mathrm{H}_{2} \mathrm{O}_{2}(30 \mathrm{wt} \%)$ in a reaction vessel at $80^{\circ} \mathrm{C}$ for 24 hours. After the reaction vessel cooled to room temperature, the Ti plates were gently rinsed three times with deionized water, dried in the ambient atmosphere, and finally calcined at $450^{\circ} \mathrm{C}$ for 1 hour to produce nanostructure-surfaced titanium. Silver was implanted into the $\mathrm{H}_{2} \mathrm{O}_{2}$-treated titanium nanostructured surface using a filtered cathodic arc plasma source. A magnetic duct with a curved shape was inserted between the plasma source and main chamber to remove macro-particles produced from the cathodic arc. The cathode rod was $10 \mathrm{~mm}$ in diameter and was made of $99.99 \%$ pure metallic silver. The Ag discharge was controlled by the main arc current between the cathode and anode. By applying a pulsed high voltage to the $\mathrm{H}_{2} \mathrm{O}_{2}-$ treated Ti samples, Ag ions were implanted; the implantation instrumental parameters are listed in Table 1. During Ag plasma immersion ion implantation (Ag-PIII), the main arc current and pulsed high voltage that was applied to the target were synchronized at a pulsing frequency of $7 \mathrm{~Hz}$. The pulse duration of the main arc current was $450 \mu \mathrm{s}$, which was the same as the pulse duration of the high voltage current.

Three different titanium surfaces were tested: untreated titanium (denoted as Ti), nanostructured titanium (NT), and Ag-implanted titanium with nanostructural surface (Ag-NT). After ultrasonic cleaning, all specimens were sterilized before the experiments began.

Surface morphologies were observed by field-emission scanning electron microscopy ([Fe-SEM] JSM-6700F; JEOL Ltd, Tokyo, Japan). The compositions of the NT and Ag-NT surfaces were determined by x-ray photoelectron spectroscopy ([XPS] PHI 5802; Physical Electronics Inc, Eden Prairie, MN).

The Ag-NT plates were soaked in $6 \mathrm{~mL} 0.9 \%$ saline solution at $37^{\circ} \mathrm{C}$ for 1 day and 3 days. At the end of incubation, the leaching liquid was collected and the amount of silver released was analyzed by inductively-coupled plasma mass spectrometry ([ICP-MS] Nu Instruments, Wrexham, UK).

\section{Determination of antimicrobial efficiency}

The antimicrobial activity of each group was tested against gram-positive $S$. mutans (UA159) bacteria, gram-negative $P g$ (ATCC33277) bacteria, and the fungus $C$. albicans (ATCC 76615). The dry pellet was rehydrated in $6 \mathrm{~mL}$ of

Table I Important instrumental parameters used in silver plasma immersion ion implantation (PIII)

\begin{tabular}{lll}
\hline & Target & Cathodic arc \\
\hline Voltage pulse duration $(\mu \mathrm{s})$ & 450 & 450 \\
Pulsing frequency $(\mathrm{Hz})$ & 7 & 7 \\
lon implantation voltage $(\mathrm{kV})$ & -15 & - \\
lon implantation time $(\mathrm{min})$ & 30 & - \\
Pressure $(\mathrm{Pa})$ & $3.6 \times 10^{-3}$ & - \\
\hline
\end{tabular}


$3.7 \%$ concentration brain heart infusion broth ([BHI], Difco, Franklin Lakes, NJ). The microbial solutions were incubated under standard anaerobic conditions $\left(80 \% \mathrm{~N}_{2}, 10 \% \mathrm{H}_{2}, 10 \%\right.$ $\mathrm{CO}_{2}$, at $\left.37^{\circ} \mathrm{C}\right)^{13,14}$ and the fungus was incubated under standard cell condition $\left(5 \% \mathrm{CO}_{2}, 95 \%\right.$ humidified air, at $\left.37^{\circ} \mathrm{C}\right)$. The second passage of microorganisms was diluted at a ratio of 1:200 in sterilized BHI and incubated. A portion of these microorganisms was then frozen in a mixture of 50\% BHI and $50 \%$ glycerol and stored at $-40^{\circ} \mathrm{C}$. All experiments were performed using these frozen stocks. Prior to the experiments, a sterile $10 \mu \mathrm{L}$ loop was used to extract a sample from the revived microbial solution and inoculate a tube containing $3 \mathrm{~mL}$ of fresh BHI.

\section{Film applicator coating assay}

A film applicator coating (FAC) assay was used to test the antimicrobial effect of each surface by directly incubating microbial cells on modified surfaces. Firstly, a solution containing microorganisms at a concentration of $1 \times 10^{8}$ colony forming units (CFUs)/mL was introduced onto the entire sample at a density of $0.05 \mathrm{~mL} / \mathrm{cm}^{2}$ and covered by polypropylene film. The samples were maintained for 1 day and re-immersed in BHI followed by vigorous vortex mixing for 5 minutes. One hundred microliter samples from the mixtures were spread on tryptic soy agar (TSA) plates and cultivated under anaerobic conditions. The active cells on plates were counted by quantifying the CFUs in accordance with the National Standards of China GB/T 4789.2 protocol.

Each test was run in triplicate and repeated on three separate occasions.

The antimicrobial effect in each group was represented by the microbial ratio, which was calculated as follows:

Microbial ratio $(\%)=(\mathrm{CFU}$ of control $-\mathrm{CFU}$ of experimental)/CFU of control $\times 100 \%$ in which $\mathrm{CFU}$ is colony-forming unit; the control group is untreated $\mathrm{Ti}$; and experimental groups are NT and Ag-NT samples.

\section{Zones of inhibition assay}

In the zones of inhibition (ZOI) assay, the concentration of strains was adjusted to $1 \times 10^{6} \mathrm{CFU} / \mathrm{mL}$ and spread on TSA plates. The dishes were incubated after samples were put on the agar plates. After 48 hours' incubation, we evaluated antimicrobial effect by quantifying the zones of inhibition.

\section{Microbial gene expressions}

The samples with microbia at Day 1 were re-immersed in BHI followed by vigorous vortex mixing for 5 minutes.
Microbes that detached from sample surfaces were collected according to the procedures specified in "Film applicator coating assay" section. Total ribonucleic acid (RNA) was extracted from the cells cultured on samples using TRIZOL reagent (Invitrogen, Carlsbad, CA). By adding chloroform to the mixture, shaking it, and centrifuging, the RNA was separated into an aqueous phase that was subsequently recovered and precipitated by equivalent isopropanol. The acquired RNA pellet was washed with $75 \%$ ethanol treated with the RNase inhibitor diethyl pyrocarbonated (DEPC) and then solubilized in sterile DEPC/water. After determining RNA concentration, the extracted RNA was reverse transcribed. Quantitative real-time polymerase chain reaction (PCR) (Bio-Rad iQ5 real-time PCR detection system; Bio-Rad Laboratories, Hercules, CA) was performed using a mixture of iQ5 SYBR ${ }^{\circledR}$ Green I supermix (Bio-Rad) and each of the forward and reverse primers and cDNA templates. The primer sequences were designed using the Primer3 web-based software. ${ }^{15}$ Design parameters were adjusted to minimize the formation of artifact products and to be able to use an annealing temperature in the PCR at approximately $60^{\circ} \mathrm{C}$, as follows: 16s rRNA, 5'-CCTACGGGAGGCAGCAGTAG-3' and 5'-CAACAGAGCTTTACGATCCGAAA3'; $G b p$ $B$ (S. mutans), 5'-ATGGCGGTTATGGACACGTT-3' and 5'-TTTGGCCACCTTGAACACCT-3'; FimA (Pg), 5'-CTGAACGAACTGCGACGCTATATGCA-3' and 5' - GT T T T T TAG T C G T T T GACGGGTCGAT-3'; Als $4,5^{\prime}$ - TCCGAGTCCATTCCAGTACTAA-3' and 5'-GTTACAGCATCACTAGAAGGAATATC-3' (C. albicans); Act1, 5'-GCCGGTGACGACGCTCCAAGAGCTG-3' and 5'-CCGTGTTCAATTGGGTATCTCAAGGTC-3'. The cycle profile included one cycle at $95^{\circ} \mathrm{C}$ for 10 seconds, 40 cycles at $95^{\circ} \mathrm{C}$ for 5 seconds, and at $60^{\circ} \mathrm{C}$ for 31 seconds. Each band intensity was quantified and normalized by reference to the $16 \mathrm{~s}$ rRNA (bacteria) and Actl (fungi) mRNA expression at Day 1.

\section{Osteoblast response to surfaces}

Primary rat osteoblasts were extracted by digesting the calvarial bone of neonatal Sprague-Dawley rats (Shanghai Laboratory Animal Center, Shanghai, China). ${ }^{16}$ The cells were cultured in Dulbecco's Modified Eagle's Medium (Gibco, Big Cabin, OK) containing 10\% fetal bovine serum (Invitrogen) and 1\% penicillin/streptomycin (Sigma-Aldrich, St Louis, MO), and incubated in a humidified atmosphere of $5 \% \mathrm{CO}_{2}$ at $37^{\circ} \mathrm{C}$. The third passage of osteoblast was used in the experiment. At subconfluence, the cells were detached and seeded onto the specimens at a density of $4 \times 10^{4}$ cells $/ \mathrm{cm}^{2}$ in the culture medium. 


\section{Cell attachment}

The samples were placed at the bottom of a 24-well plate and then osteoblast cell suspensions at a density of $4 \times 10^{4}$ cells $/ \mathrm{cm}^{2}$ were seeded on to the substrates and incubated in a $5 \% \mathrm{CO}_{2}$ incubator for 1 day. After the incubation period, the samples were washed twice with a phosphate-buffered saline solution (PBS) before being fixed with 3\% glutaraldehyde for at least 2 hours at $4{ }^{\circ} \mathrm{C}$. The samples were subjected to step dehydration with serial ethanol for 10 minutes each, and then coated with platinum for imaging using an SEM (JEOL).

\section{In vitro cell viability assay}

The confluent osteoblast cultures (passage three) were detached from the culture flask by incubation with $0.1 \%$ trypsin and $0.1 \%$ ethylenediaminetetraacetic acid (EDTA) for 5 minutes. The solution of osteoblasts was centrifuged at $1000 \mathrm{~g}$ for 5 minutes, and re-suspended in the medium. The viability of cells colonizing on the samples was evaluated by 3-(4,5)-dimethylthiahiazo(-z-y1)-3, 5-diphenytetrazoliumromide (MTT; Sigma-Aldrich) reduction assay. The positive control in this experiment was cells cultured in $5 \mathrm{~g} / \mathrm{L}$ phenol solution. After 1 day and 3 days, $1 \mathrm{~mL}$ of $0.5 \%$ MTT solution was added and the plate was incubated for 4 hours at $37^{\circ} \mathrm{C}$ in a humidified $5 \% \mathrm{CO}_{2}$ atmosphere. The solution was subsequently removed and samples with osteoblasts were transferred to new wells. Dimethyl sulfoxide (DMSO) $200 \mu \mathrm{L}$ was added to each well, and the plate was shaken for 15 minutes before measuring absorbance at $570 \mathrm{~nm}$ (the reference value was $630 \mathrm{~nm})$.

\section{Osteogenic gene expression}

To investigate the influence of various modified substrates on the gene expression of seeded osteoblasts, the total RNA from osteoblasts grown on various substrates for 3 and 7 days was isolated using a TRIzol ${ }^{\circledR}$ reagent (Invitrogen) according to the manufacturer's protocol. The gene expression of osteoblast differentiation markers - osteocalcin $(O c n)$, alkaline phosphatase (Alp), and runt-related transcription factor 2 (RunX2) - were evaluated by realtime polymerase chain reaction (PCR). Glyceraldehyde 3-phosphate dehydrogenase (Gapdh) was utilized as an endogenous housekeeping gene. PCR conditions included an initial denaturation at $95^{\circ} \mathrm{C}$ for 10 minutes, followed by a 40 cycle amplification consisting of denaturation at $95^{\circ} \mathrm{C}$ for 15 seconds, and annealing at $60^{\circ} \mathrm{C}$ for $30 \mathrm{sec}-$ onds. The sequences of primers were designed and were as follows:
Alp: 5'CGGAAGTGAGGCAGGTAG3',

5'AGAGCCCACAATGGACAG3'

Ocn: 5'GTGCCGTCCATACTTTCG3',

5'GACCACATTGGCTTCCAG3'

RunX2: 5'GCTTCTCCAACCCACGAATG3', 5'GAACTGATAGGACGCTGACGA3'

Gapdh: 5'TGCTGGGGCTGGCATTGCTC3', 5'CCCCAGGCCCCTCCTGTTGT3'

Quantification of gene expression was based on the $\mathrm{Ct}$ (threshold cycle) value for each sample calculated as the average of three replicate measurements. The relative expression levels for each gene of interest were normalized to that of Gapdh.

\section{Statistical analysis}

The assays were performed in triplicate and the data were expressed as means \pm standard deviations. Each experiment was repeated three times. A one-way analysis of variation (ANOVA) combined with a Student-Newman-Keuls post hoc test was utilized to determine the level of significance. $P<0.05$ was regarded to be significant and $P<0.01$ was considered highly significant.

\section{Results}

\section{Surface characterization and silver release}

The surface SEM views of Ti, NT, and Ag-NT samples are shown in Figure 1. Compared with the original surface of titanium (Figure 1A), the NT has a typical nanosheet structure (Figure 1B). After Ag implantation, the nanosheets on the titanium surface were damaged but still retained the

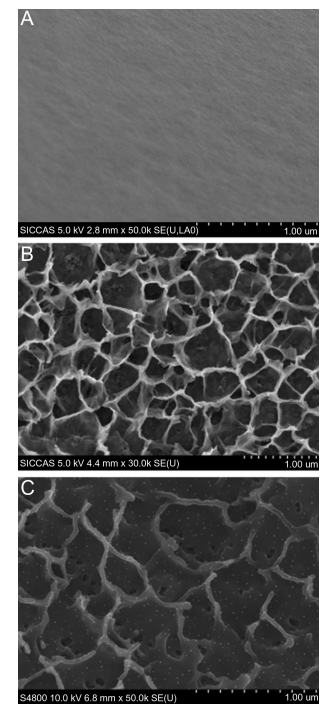

Figure I Surface SEM views of Ti (A), NT (B), and Ag-NT (C) samples. Note: Scale bar $=I \mu \mathrm{m}$.

Abbreviation: SEM, scanning electron microscopy. 
nanosheet structure with some Ag nanoparticles of about $10 \mathrm{~nm}$, as shown in Figure 1C. From the visual field of SEM, these nanoparticles were homogeneously distributed. The XPS full spectrum acquired from NT and Ag-NT surfaces further confirm the existence of Ti and $\mathrm{O}$ on NT, and Ag, and Ti and $\mathrm{O}$ on Ag-NT, as shown in Figure 2A and B. The content of Ag in the outmost layer of Ag-NT was calculated from the XPS spectrum and is approximately $3.5 \%$. Figure $2 \mathrm{C}$ shows the XPS $\mathrm{Ag} 3 \mathrm{~d}$ spectrum obtained from the Ag-NT surface. The Ag 3d doublet at $374.05 \mathrm{eV}(\mathrm{Ag} \mathrm{3d} 3 / 2)$ and $368.04 \mathrm{eV}(\mathrm{Ag} \mathrm{3d5/2})$ corresponds to metallic silver, indicating that the nanoparticles are metallic silver. Additionally, the silver release in saline was evaluated by ICP-MS. Less than $10 \mathrm{ppb} \mathrm{Ag}$ was detected in the Ag-NT after soaking in saline for 3 days, indicating the limited leaching of silver from the Ag-NT surfaces.

\section{Microbial response to different surfaces Antimicrobial efficiency}

The microorganisms attached to the surfaces of Ti, NT, and Ag-NT were collected and re-cultivated on agar according to the FAC assay. Figure 3A-C shows typical re-cultivated microbial colonies. A large amount of microorganisms occupied the Ti surface while relatively few appeared on the NT and Ag-NT surfaces. The number of CFU after 1 day (Figure 3D) agrees with the colonies on agar (Figure $3 \mathrm{~A}-\mathrm{C}$ ). Table 2 shows

A

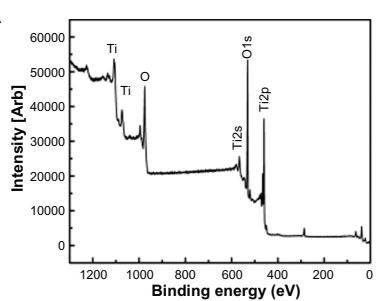

B

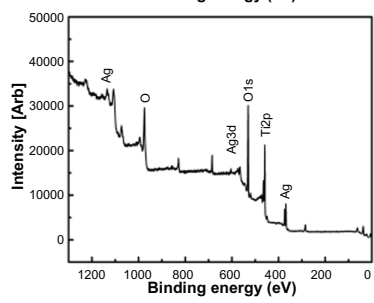

C

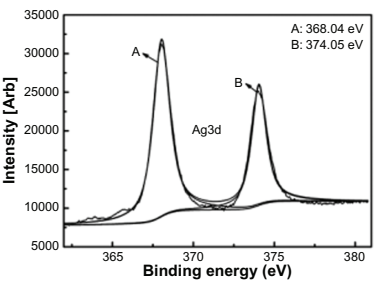

Figure 2 XPS full spectra of NT (A) and Ag-NT (B) surfaces, XPS Ag 3d spectrum of Ag-NT surface (C).

Abbreviation: XPS, x-ray photoelectron spectroscopy.
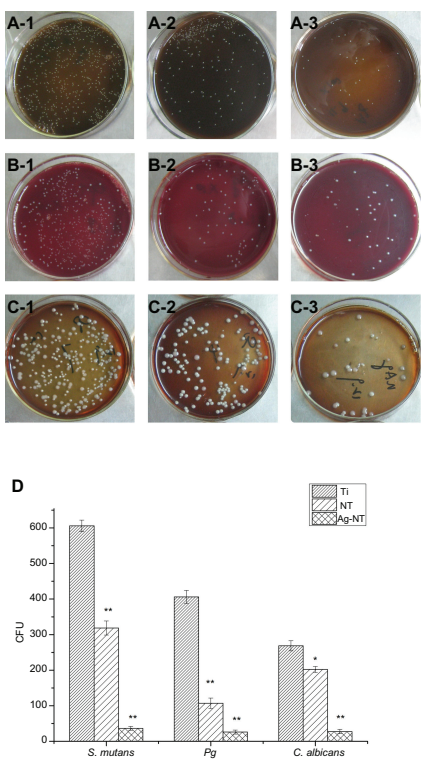

Figure 3 In FAC assay, re-cultivated microbial colonies on TSA : S. mutans colonies are collected from Ti (A-I), NT (A-2), and Ag-NT (A-3). The Pg colonies are collected from $\mathrm{Ti}$ (B-1), NT (B-2), and Ag-NT (B-3). C. albicans colonies are collected from Ti (C-I), NT (C-2), and Ag-NT (C-3); Counts of CFU in FAC assay (D). One-way ANOVA followed by SNK was utilized to determine the level of significance.

Notes: $* P<0.05 ; * * P<0.01$

Abbreviations: ANOVA, analysis of variation; CFU, colony forming unit; FAC, film applicator coating; SNK, Student-Newman-Keuls; TSA, tryptic soy agar.

the antimicrobial ratio of the modified surfaces, and this data allows the calculation of antimicrobial ratios of different titanium surfaces to these three microbes. The results indicate that Ag-NT is able to reduce strains with the antimicrobial ratios of $93.99 \%$ (S. mutants), $93.57 \%(P g)$ and $89.78 \%$ (C. albicans), while NT has the ratios of $47.45 \%, 73.61 \%$, and $24.77 \%$, respectively. However, ZOI tests do not show any difference among the three groups. It is believed that the antimicrobial ability of Ag-NT depends on the amount of silver being released. ${ }^{17}$ However, in our study, since leaching silver from the surface is limited, Ag-NT acts through direct contact, rather than silver ions being released. Interestingly, NT also has some antimicrobial ability, although it appears much lower than that of Ag-NT.

\section{Microbial gene expression}

Quantitative real-time PCR analysis of the levels of mRNA in cells grown on different surfaces after 1 day of culturing are shown in Figure 4. The $G b p B$ mRNA expression on

Table 2 Antimicrobial ratio of the modified surfaces. Ag-NT exhibited a stronger effect than NT

\begin{tabular}{lll}
\hline Antimicrobial ratio (\%) & NT group & Ag-NT group \\
\hline S. mutans & $47.45 \pm 2.67$ & $93.99 \pm 8.99$ \\
Pg & $73.61 \pm 5.89$ & $93.57 \pm 7.98$ \\
C. albicans & $24.77 \pm 1.9$ & $89.78 \pm 6.8$ \\
\hline
\end{tabular}




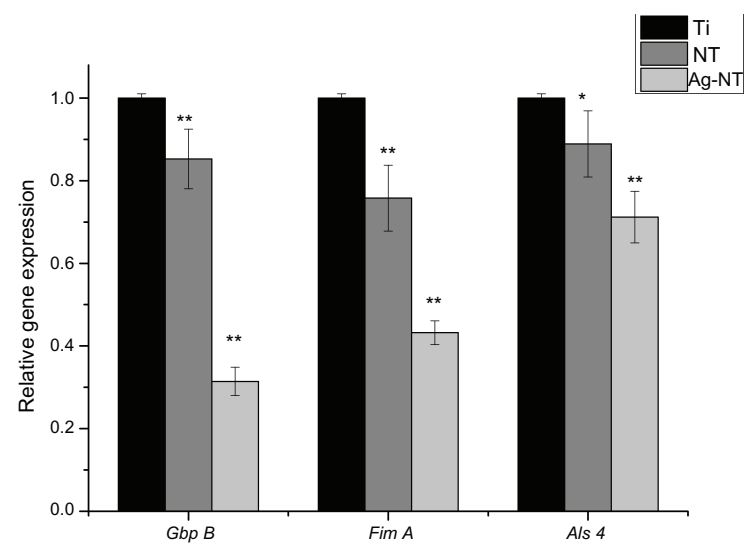

Figure 4 Quantitative real-time PCR analysis of the levels of microbial genes of mRNA in cells grown on different surfaces after I day of culturing.

Notes: Values are the mean \pm SD of three measurements; $* P<0.05 ; * * P<0.0$ I.

Abbreviations: PCR, polymerase chain reaction; SD, standard deviation.

NT and Ag-NT surfaces was lower than that on Ti at 1 day (0.85 0.31-fold). Similarly, the mRNA expressions of FimA and Als 4 on NT and Ag-NT were lower than those on Ti. This means that the expressions of genes can be reduced significantly on the Ag-NT and NT groups, which confirms that these have antimicrobial activity. The surfaces with silver nanoparticles display high antimicrobial activity against oral microorganisms. For C. albicans, however, it showed a weaker effect.

\section{Osteoblastic response to different surfaces}

\section{Osteoblast adhesion on surfaces}

Figure 5 shows the SEM images of Ti, NT, and Ag-NT surfaces seeded with osteoblast cells for 1 day. The Ti surface supported osteoblast cell attachment, with the cells cultured for 24 hours showing spreading (Figure 5A and A-1). The SEM images of the NT and Ag-NT surfaces (Figure 5B and $\mathrm{C}$ ) show that cells were able to attach and grow on the surfaces. It can be seen that the cells in Figure 5B-1 and C-1 tended to spread extensively and clump together.
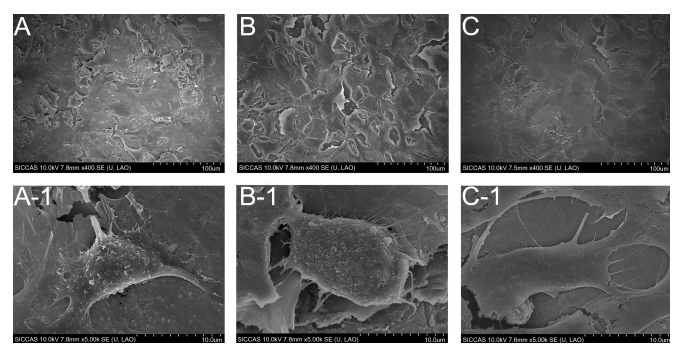

Figure 5 SEM images of different surfaces after exposure to osteoblast cell suspension $\left(4 \times 10^{4}\right.$ cells $\left./ \mathrm{cm}^{2}\right)$ for I day: (A) untreated Ti surface; (B) NT surface; (C) Ag-NT surface; under higher magnification, (A-I and B-I), and (C-I) show the morphology of adhered osteoblasts on Ti, NT, and Ag-NT, respectively.

Abbreviation: SEM, scanning electron microscopy.

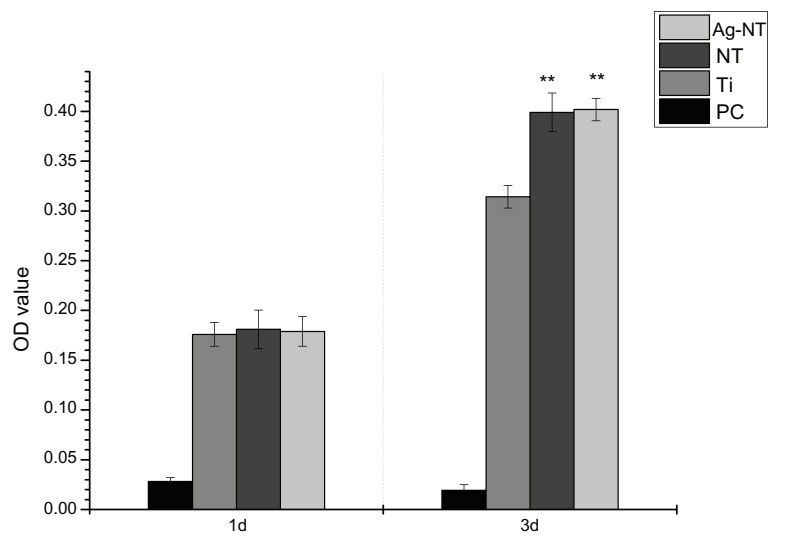

Figure 6 Osteoblasts viability assay after I and 3 days incubation. Among groups, there were no significant differences observed at day I, while the $\mathrm{Ti}$ group was lower than modified surfaces at day 3. PC is always very low.

Notes: Means $\pm S D$; $* P<0.05 ; * * P<0.01$.

Abbreviations: PC, positive control; OD, optical density; SD, standard deviation.

\section{Cytocompatibility assay}

Osteoblast viability was evaluated after 1 and 3 days of incubation. The results are shown in Figure 6. According to the absorbance values on different surfaces, NT and Ag-NT are equivalent $(P>0.05)$ at 3 days of culture, while Ti is significantly lower $(P<0.01)$. However, there was no significant difference $(P>0.05)$ in the viability of cells on the three samples after 1 day of culture. It appears that Ag-NT and NT have little cytotoxicity, but rather have more favorable effects on osteoblasts.

\section{Osteogenic gene expression}

The levels of osteogenic phenotype gene expression are shown in Figure 7. The mRNA expression levels of Alp, RunX2, and $O C N$ increased with incubation time in all groups. After 3 days of culturing, the expression of Alp on NT was significantly higher $(P<0.01)$ than those on Ag-NT and Ti, while the expression of Ocn did not differ among them $(P>0.01)$. After 7 days of culturing, the expressions of both Alp and Ocn on the NT and Ag-NT surfaces were higher than those on Ti $(P<0.01)$. Additionally, RunX2 mRNA expression was higher in cells grown on NT and Ag-NT surfaces than that on Ti surface at 3 days. At 7 days, there was no difference $(P>0.01)$ between all of them in the expression of $R u n X 2$.

\section{Discussion}

There is significant research being performed on the prevention of implant-associated infection. ${ }^{1,8}$ Although many antimicrobial coatings have been studied, most of them are prone to degrade in physical environment quickly. Many coatings also depend on the release of antimicrobial agents 

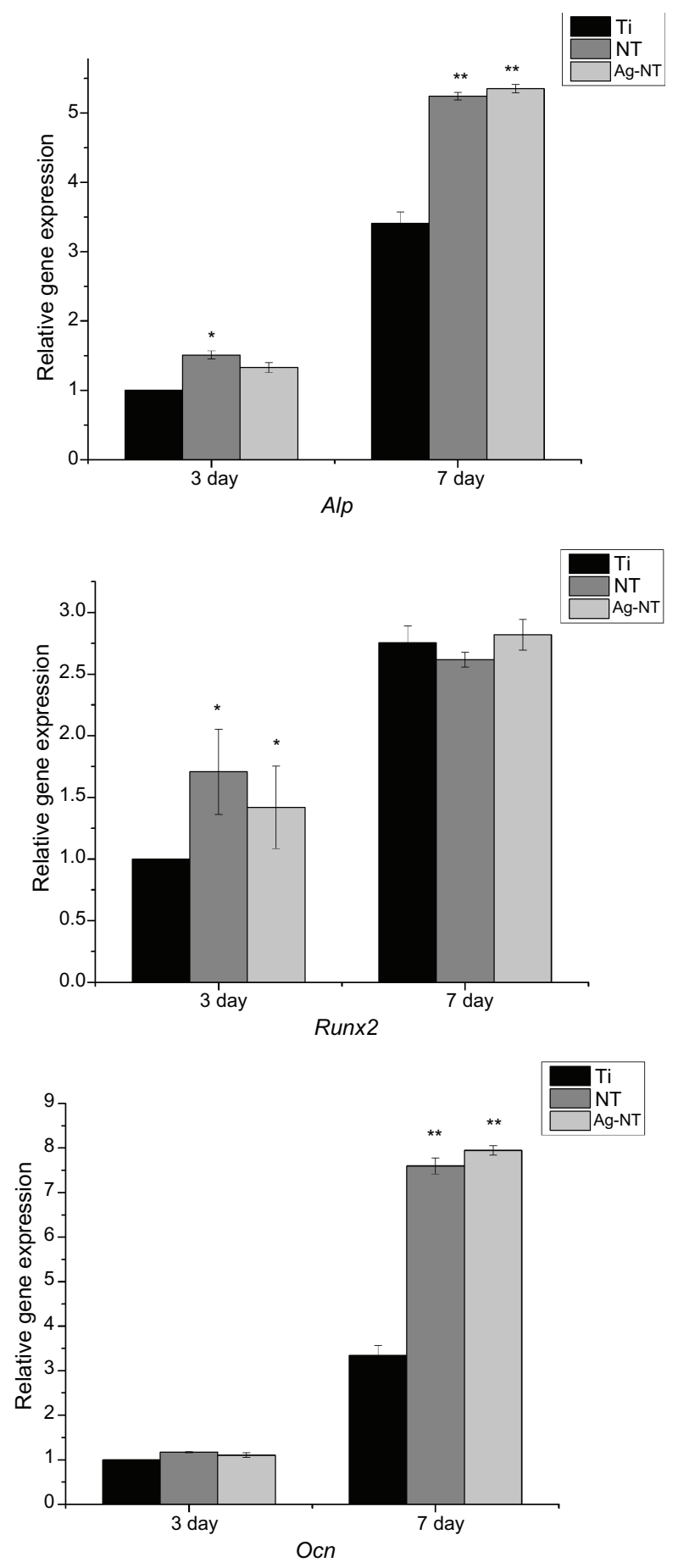

Figure 7 Quantitative real-time PCR analysis of the mRNA for alkaline phosphatase $(A / p)$, runt-related transcription factor 2 (Runx2), and osteocalcin $(O \mathrm{cn})$ in osteoblast cells grown on different surfaces after 3 and 7 days of culturing.

Notes: Values are the mean \pm SD of three measurements; $* P<0.05 ; * * P<0.01$. Abbreviations: PCR, polymerase chain reaction; SD, standard deviation.

such as antibiotics, ${ }^{4,5}$ which may cause safety concerns regarding release rates and antibiotic resistance. Therefore, implant surfaces should ideally have antimicrobial properties without releasing antibiotic agents at too fast a rate, while also being beneficial for osteogenesis. In this study,
Ag-implanted titanium with a nanostrucured surface (Ag-NT) was developed and, as is shown in Figure $1 \mathrm{C}$, the nanosheets on the Ag-NT surface retained their morphology with $10 \mathrm{~nm}$ $\mathrm{Ag}$ nanoparticles being homogeneously distributed. The Ag $3 \mathrm{~d}$ doublet at $374.05 \mathrm{eV}(\mathrm{Ag} \mathrm{3d3/2})$ and $368.04 \mathrm{eV}(\mathrm{Ag} \mathrm{3d5/2)}$ corresponds to metallic silver, indicating that the nanoparticles are metallic silver.

To determine the antimicrobial effect of titanium surfaces, the viability of bacteria was tested according to FAC and ZOI assays. ZOI relies on the leaching of silver ions from the material's surface and inhibiting the growth of the bacteria around the materials, while FAC examines the effect of direct contact. ${ }^{18}$ In our FAC test, Ag-NT exhibited the strongest antimicrobial effect, with antimicrobial ratios of almost 90\% (Table 2). However, the ZOI test exhibited no zone of inhibition. The ICP-MS detected only a small quantity of silver ions being leached into an aqueous environment. These results indicate that only microorganisms that come directly into contact with surfaces can be affected by the antimicrobial effects of Ag-NT. This does, however, mean that silver ions will not accumulate in tissues around implants, which could otherwise result in cytotoxicity or other side effects.

The antimicrobial ratio of C. albicans (Table 2) was lower than those of the bacteria $S$. mutans and $P g$. This may because this fungus has more organs and is larger, which may increase its resistance to the silver. Interestingly, NT also reduced the microbial colonies (Figure 3 ) to some extent. Rough or textured dental implant surfaces have been shown to enhance bacterial and fungal colonization, ${ }^{19,20}$ but the finding of the current study is consistent with the work of Sabrina et al, ${ }^{18}$ who found that nanorough $\mathrm{Ti}$ decreased the adherence of Staphylococcal aureus, Staphylococcus epidermidis, and Pseudomonas aeruginosa. ${ }^{20}$ It is believed that the increased surface energy of a nanorough surface leads to greater protein adsorption, ${ }^{21}$ and results in decreased bacterial attachment. ${ }^{22,23}$ It was also found that direct contact of the bacteria (E. coli and $S$. aureus) with the extremely sharp edges of the nanowalls could damage bacterial cell membranes. ${ }^{24,25}$ The FAC test performed in the current study (Figure 3) showed that fewer active bacteria adhered to NT than to Ti. Furthermore, the antimicrobial mechanism of NT and Ag-NT surfaces was explored at the genetic level.

Some researchers have suggested that silver nanoparticles provide an extremely large surface area for interaction with microorganisms, with this interaction coming from all directions, and that the nanoparticles can attach to and penetrate the cell membrane. ${ }^{26-28}$ Others have demonstrated that silver nanoparticles can cause bacterial inactivation 
and prevent bacterial replication in vitro by binding to DNA and to the sulfhydryl groups of the metabolic enzymes in the bacterial electron transport chain. ${ }^{29}$ These researches can represent the structural changes in bacteria during the process of bacterial demolishment. However, changes of microbial relative genes expressions may take place earlier than morphological alteration, and it may be more objective and sensitive than the microscopic view.

Our data (Figure 4) suggests that Gbp B in S. mutans, Fim $A$ in $P g$, and Als 4 in C. albicans were all reduced on modified surfaces. The tendency was in good agreement with the FAC result. The Ag-NT showed a strong and consistent antimicrobial ability against both bacteria and fungi. Since Gbp B in $S$. mutans may have an important role in cell wall construction, this may also be important in cell separation and cell wall maintenance. ${ }^{30,31} \mathrm{~A}$ decrease of $G b p B$ may indicate the destruction of the cell wall and cause cell separation of $S$. mutans. Fim $A$ in $P g$ plays a key role in bacterial adhesion and periodontal invasion. ${ }^{32}$ The Ag-NT surface may reduce $P g$ adhesion by inhibiting the expression of Fim A. Als 4 is an important gene for adhesion in C. albicans. ${ }^{33}$ The reduction of the expression of Als 4 means that the adhesive ability of $C$. albicans will have been reduced. However, gram-positive bacteria, gram-negative bacteria, and fungi may have slightly different mechanisms. As in our research, silver nanoparticles could damage the microbial cell-wall structure or cause cell separation, and inhibit the adhesive abilities by preventing gene expression. Any changes in gene expressions may help illuminate the mechanisms of the antimicrobial properties that are seen. Though there are limited reports about the antimicrobial effect of the nanostructure of materials, the results of the current research suggest that nanosheets may be effective in reducing related gene expression, possibly by damaging the cell wall, effecting cell separation, or altering the organism's adhesive ability. This may also explain the results of the FAC test in which NT showed some antimicrobial properties.

This research also tested modified surfaces for cytocompatibility and osteogenesis. ${ }^{6,33}$ During the short period of testing, NT and Ag-NT did not show any cytotoxicity to osteoblasts through direct contact. At 3 days, the number of cells on the surfaces of NT and Ag-NT were higher than that on Ti, which suggests that Ag-NT has no adverse effect toward osteoblasts, and may slightly promote their growth. Although the cytocompatibility of silver nanoparticles in human body is still debated, the current study did not find any indication of cytotoxicity. According to the SEM images, the number of osteoblasts adhered to the Ti surface after 1 day was similar to those on NT and Ag-NT (Figure 5A-C). However, it was found that osteoblasts on NT and Ag-NT spread more extensively and had more pseudopodial protrusions and microvilli extending from the cell body (Figure 5A-1-C-1). This implies that cell-surface and cell-cell interactions are much stronger on NT and Ag-NT, which could be attributed to the nanostructure properties of these surfaces. ${ }^{34}$ Since bone tissues are composed of hierarchical nanocomposites, the proper nanotopography can promote osteogenesis from the biomimetic viewpoint. ${ }^{35}$ In this work, osteoblasts specializing in mineralization have been evaluated by enhancing the expression of three gene types of osteogenic markers (Alp, RunX2, and $O c n$ ) in adherent cells to the titanium surfaces. Alp and Ocn play crucial roles as phenotypic markers for early and later stage bone formation, and increased Alp and $O c n$ expression correlates with increased bone formation. ${ }^{36}$ As well as this, $R u n X 2$ has been identified as the primary transcription factor for osteoblastic differentiation. ${ }^{37}$ In vitro studies have shown that RunX2 positively controls Ocn expression. ${ }^{38} \mathrm{NT}$ enhances the expression of Alp, Ocn, and RunX2, as well as the Ag-NT samples, since NT and Ag-NT surfaces can raise the osteogenic marker's expression and result in better osteogenesis. Previous research has also demonstrated that nanorough, nanotubular, and nanotextured Ti can enhance osteoblast adhesion and other functions (such as alkaline phosphatase synthesis, calcium deposition, and collagen secretion) compared to their micron or smooth counterparts. ${ }^{39}$

It appears that a nanostructured surface with silver nanoparticles incorporated (Ag-NT) may be a better choice for dental implants than nanostructured titanium (NT), since silver nanoparticles possess stronger bactericidal ability which may be effective in the oral environment. Neither material appears to impair osteoblasts, and they may enhance osteogenic gene expression. The antimicrobial activity of these modified titanium surfaces should be further investigated in animal experiments.

\section{Conclusion}

In this work, Ag was implanted into titanium with a nanostructured surface. This Ag-implanted titanium not only showed a strong antimicrobial effect against oral microorganisms including $S$. mutans (UA 159), Pg (ATCC 33277), and C. albicans (ATCC 76615), but also had appeared to promote osteogenesis with increased cell attachment, viability, and osteogenic gene expression (Alp, 
RunX2, and $O c n)$. This study suggests that Ag-implanted titanium with a nanostructured surface is a good material for dental implants due to its antimicrobial properties and osteogenic effect.

\section{Acknowledgments}

Joint financial support was received from the National Basic Research Program of China (973 Program, 2012CB933601), National Natural Science Foundation of China (31100675 and 51071168), and Shanghai Leading Academic Discipline Project (S30206).

\section{Disclosure}

The authors report no conflicts of interest in this work.

\section{References}

1. Geetha M, Singh AK, Asokamani R, Gogia AK. Ti based biomaterials, the ultimate choice for orthopaedic implants - a review. Prog Mater Sci. 2009;54(3):397-425.

2. Esposito M, Hirsch J, Lekholm U, Thomsen P. Biological factors contributing to failures of osseointegrated oral implants. (II). Etiopathogenesis. Eur J Oral Sci. 1998;106(3):721-764.

3. Monteiro DR, Gorup LF, Takamiya AS, Ruvollo-Filho AC, de Camargo ER, Barbosa DB. The growing importance of materials that prevent microbial adhesion: antimicrobial effect of medical devices containing silver. Int J Antimicrob Agents. 2009;34(2):103-110.

4. Antoci V, Adams JCS, Parvizi J, et al. The inhibition of Staphylococcus epidermidis biofilm formation by vancomycin modified titanium alloy and implications for the treatment of periprosthetic infection. Biomaterials. 2008;29(35):4684-4690.

5. Vester H, Wildemann B, Schmidmaier G. Gentamycin delivered from a PDLLA coating of metallic implants: in vivo and in vitro characterisation for local prophylaxis of implant-related osteomyelitis. Injury. 2010;41(10):1053-1059.

6. Agarwal A, Weis TL, Schurr MJ, et al. Surfaces modified with nanometerthick silver-impregnated polymeric films that kill bacteria but support growth of mammalian cells. Biomaterials. 2010;31(4):680-690.

7. Chen X, Schluesener HJ. Nanosilver: a nanoproduct in medical application. Toxicol Lett. 2008;176(1):1-12.

8. Cao H, Liu X, Meng F, et al. Biological actions of silver nanoparticles embedded in titanium on antibacterial activity in vitro. Biomaterials. 2011;32(3):693-705.

9. Mombelli A, Décaillet F. The characteristics of biofilms in peri-implant disease. J Clin Periodontol. 2011;38(Suppl 11):203-213.

10. Bürgers R, Hahnel S, Reichert TE, et al. Adhesion of Candida albicans to various dental implant surfaces and the influence of salivary pellicle proteins. Acta Biomater. 2010;6(6):2307-2313.

11. Mendonça G, Mendonça DB, Aragão FJ, Cooper LF. Advancing dental implant surface technology - from micron- to nanotopography. Biomaterials. 2008;29(28):3822-3835.

12. Yao C, Slamovich EB, Webster TJ. Enhanced osteoblast functions on anodized titanium with nanotube-like structures. J Biomed Mater Res A. 2008;85(1):157-166

13. Shibata Y, Suzuki D, Omori S, et al. The characteristics of in vitro biological activity of titanium surfaces anodically oxidized in chloride solutions. Biomaterials. 2010;31(33):8546-8555.

14. Omori S, Shibata Y, Arimoto T, Igarashi T, Baba K, Miyazaki T. Microorganism and cell viability on antimicrobially modified titanium. J Dent Res. 2009;88(10):957-962.
15. Rozen S, Skaletsky H. Primer3 on the WWW for general users and for biologist programmers. Methods Mol Biol. 2000;132:365-386.

16. Liao J, Zhu Z, Mo A, Li L, Zhang J. Deposition of silver nanoparticles on titanium surface for antibacterial effect. Int J Nanomedicine. 2010;5:261-267.

17. Zhao L, Wei Y, Li J, Han Y, Ye R, Zhang Y. Initial osteoblasts functions on Ti-5Zr-3Sn-5Mo-15Nb titanium alloy surfaces modified by microarc oxidation. J Biomed Mater Res A. 2010;92(2):432-440.

18. Sabrina DP, Erik T, Theresa R, Thomas JW. The relationship between the nanostructure of titanium surfaces and bacterial attachment. Biomaterials. 2010;31:706-713.

19. Quirynen M, van der Mei HC, Bollen CM, et al. An in vivo study of the influence of the surface roughness of implants on the microbiology of supra- and subgingival plaque. J Dent Res. 1993;72(9):1304-1309.

20. Khang D, Kim SY, Liu-Snyder P, Palmore GT, Durbin SM, Webster TJ. Enhanced fibronectin adsorption on carbon nanotube/poly(carbonate) urethane: independent role of surface nano-roughness and associated surface. Biomaterials. 2007;28(32):4756-4768.

21. Anagnostou F, Debet A, Pavon-Djavid G, Goudaby Z, Helary G, Migonney V. Osteoblast functions on functionalized PMMA-based polymers exhibiting Staphylococcus aureus adhesion inhibition. Biomaterials. 2006;27(21):3912-3919.

22. Kinnari TJ, Peltonen LI, Kuusela P, Kivilahti J, Kononen M, Jero J. Bacterial adherence to titanium surface coated with human serum albumin. Otol Neurotol. 2005;26(3):380-384.

23. Rai M, Yadav A, Gade A. Silver nanoparticles as a new generation of antimicrobials. Biotechnol Adv. 2009;27(1):76-83.

24. Akhavan O, Ghaderi E. Toxicity of graphene and graphene oxide nanowalls against bacteria. ACS Nano. 2010;4(10):5731-5736.

25. Akhavan O, Ghaderi E. Copper oxide nanoflakes as highly sensitive and fast response self-sterilizing biosensors. J Mater Chem. 2011;21(34):12935-12940.

26. Jung WK, Koo HC, Kim KW, Shin S, Kim SH, Park YH. Antibacterial activity and mechanism of action of the silver ion in Staphylococcus aureus and Escherichia coli. Appl Environ Microbiol. 2008;74(7): 2171-2178.

27. Li W, Xie X, Shi Q, Duan S, Ou-Yang Y, Chen Y. Antibacterial activity and mechanism of silver nanoparticles on Escherichia coli. Appl Microbiol Biotechnol. 2010;85(4):1115-1122.

28. Darouiche RO. Anti-infective efficacy of silver-coated medical prostheses. Clin Infect Dis. 1999;29(6):1371-1377.

29. Fujita K, Matsumoto-Nakano M, Inagaki S, Ooshima T. Biological functions of glucan-binding protein B of Streptococcus mutans. Oral Microbiol Immunol. 2007;22(5):289-292.

30. Chia JS, Chang LY, Shun CT, Chang YY, Tsay YG, Chen JY. A 60-kilodalton immunodominant glycoprotein is essential for cell wall integrity and the maintenance of cell shape in Streptococcus mutans. Infect Immun. 2001;69(11):6987-6998.

31. Ichiro N, Atsuo A, Masae K, Nakamura T, Kawabata S, Hamada S. Functional differences among FimA variants of Poephyromonas gingivalis and their effects on adhesion to and invasion of human epithelial cells. Infect Immun. 2008;70(1):277-285.

32. Qi Q, Hu T, Fu C, Zhou X. Comparison of ALS gene mRNA between sessile and planktonic Candida albicans of oral cavities. Hua Xi Kou Qiang Yi Xue Za Zhi. 2005;23(3):233-236. Chinese.

33. Ramstedt M, Ekstrand-Hammarström B, Schukarev AV, et al Bacterial and mammalian cell response to poly(3-sulfopropyl methacrylate) brushes loaded with silver halide salts. Biomaterials. 2009;30(8):1524-1531.

34. Puckett S, Pareta R, Webster TJ. Nano rough micron patterned titanium for directing osteoblast morphology and adhesion. Int J Nanomedicine. 2008;3(2):229-241.

35. Zhao L, Wang H, Huo K, et al. Antibacterial nanostructured titania coating incorporated with silver nanoparticles. Biomaterials. 2011;32(24):5706-5716. 
36. Walsh S, Jefferiss C, Stewart K, Jordan GR, Screen J, Beresford JN. Expression of the developmental markers STRO-1 and alkaline phosphatase in cultures of human marrow stromal cells: regulation by fibroblast growth factor (FGF)-2 and relationship to the expression of FGF receptors 1-4. Bone. 2000;27(2):185-195.

37. Komori T, Yagi H, Nomura S, et al. Targeted disruption of $\mathrm{Cbfa} 1$ results in a complete lack of bone formation owing to maturational arrest of osteoblasts. Cell. 1997;89(5):755-764.
38. Ducy P, Zhang R, Geoffroy V, Ridall AL, Karsenty G. Osf2/Cbfa 1: a transcriptional activator of osteoblast differentiation. Cell. 1997;89(5):747-754.

39. Yao C, Slamovich EB, Webster TJ. Enhanced osteoblast functions on anodized titanium with nanotube-like structures. J Biomed Mater Res A. 2008;85(1):157-166.

\section{Publish your work in this journal}

The International Journal of Nanomedicine is an international, peerreviewed journal focusing on the application of nanotechnology in diagnostics, therapeutics, and drug delivery systems throughout the biomedical field. This journal is indexed on PubMed Central, MedLine, CAS, SciSearch ${ }^{\circledR}$, Current Contents ${ }^{\circledR} /$ Clinical Medicine,
Journal Citation Reports/Science Edition, EMBase, Scopus and the Elsevier Bibliographic databases. The manuscript management system is completely online and includes a very quick and fair peer-review system, which is all easy to use. Visit http://www.dovepress.com/ testimonials.php to read real quotes from published authors.

Submit your manuscript here: http://www.dovepress.com/international-journal-of-nanomedicine-journal 